\title{
Quality of care for sexually transmitted infections (STIS) syndromic case management at public health facilities of gamo gofa zone, Southern Ethiopia
}

\begin{abstract}
Background: Sexually Transmitted Infections (STIs) and their associated complications are regarded as one of the top five reasons for which adults avail themselves for health care services in developing countries.

Objective: This is study was aimed to assess quality of care for STIs Syndromic case management at public health facilities of Gamo Gofa Zone, Southern Ethiopia.

Methods: Facility based cross sectional study that used both quantitative and qualitative methods of data collection was conducted from Jan 1 to Feb 28, 2015. The study included 10 health centers and 2 hospitals selected by simple random sampling, their respective 12 heads, 250 clinicians selected by simple random sampling from the health facilities after proportional allocation to size and 26 mystery clients. The data were entered into EPI info version 7.0.1 and exported to SPSS version 20 for analysis.

Results: Overall there were no STI Syndromic management guidelines in all health facilities, only $12.8 \%$ of clinicians have taken training on Syndromic management of STIs, highest knowledge of clinicians was of urethral discharge (27.2\%). Profession of clinicians and type of health facility $(\mathrm{AOR}=0.194 ; 95 \% \mathrm{CI}=0.092,0.412)$ were determinants of urethral discharge knowledge. Only in $8.3 \%$ of cards did the clinicians correctly follow the guideline. Only $11.54 \%$ of mystery clients were satisfied with the services and only $15.38 \%$ will recommend the provider to a friend.
\end{abstract}

Conclusion: The quality of care for STIs Syndromic case management at public health facilities of Gamo Gofa Zone was poor.

Keywords: STI, std, HIV/aids, syndromic management, quality care, mystery client, gamo gofa zone, gonorrhea, chlamydial infection, syphilis, trichomoniasis, chancroid, genital herpes, hepatitis b
Volume 6 Issue I - 2017

\author{
Addisu Alemayehu Gube, 'Wanzahun \\ Godana $^{2}$ \\ 'Department of Nursing, College of Medicine and Health \\ Sciences, Arba Minch University, Ethiopia \\ ${ }^{2}$ Department of Public Health, College of Medicine and Health \\ Sciences, Arba Minch University, Ethiopia
}

Correspondence: Addisu Alemayehu Gube, Arba Minch University, Ethiopia, Tel +251912119726, Fax +251468810279, Email addis 166@gmail.com

Received: April 08, 2017| Published: June 14, 2017

\section{Introduction}

Sexually transmitted infections (STIs) are infections that are mainly transmitted from person-to-person through sexual contact. Almost 30 types of causative bacteria, viruses and parasites have been isolated, which are responsible for multiple STDs such as gonorrhea, chlamydial infection, syphilis, trichomoniasis, chancroid, genital herpes, HIV infection and hepatitis B infection. ${ }^{1,2}$ They are important because of their magnitude, potential complications and their interaction with HIV/AIDS. ${ }^{3-6}$ Even though there is little information on the incidence and prevalence of STIs in Ethiopia, the problem of STIs is generally believed to be similar to other developing countries. ${ }^{4,7}$ The aim of Syndromic approach is to identify and treat a syndrome with combination therapy which will also take care of the main causative pathogen. Ethiopia has been promoting Syndromic approach since 2001 by adopting the WHO generic guide lines to serve as a national guideline for the management of STIs: since then, trainings on Syndromic approach have been given for health care workers. ${ }^{4,8-10}$ The complications caused by failure to diagnose and treat STIs include infertility, congenital abnormality, adverse neurological condition, cardiovascular risks, ectopic pregnancy, anogenital cancer and even premature death of neonates to mention but a few. ${ }^{11,12}$ One means of reducing the impact of STIs is the management of STIs using the Syndromic approach. ${ }^{4}$ Many factors play a part in the successful control of STIs, including availability of effective and affordable drugs, accessible and acceptable health services, training and supervision of healthcare workers, and behavioral interventions to prevent new infections by promoting safer sex..$^{13}$ Quality is measurable. A system is usually made up of three components: input(structure), process and output/outcome. ${ }^{14,15}$ In order to provide quality care for sexually transmitted infections, Ethiopia has been giving training on Syndromic approach for health professionals but whether the professionals are working accordingly or not need to be explored. Therefore, the purpose of this study is to assess quality of care for sexually transmitted infections Syndromic case management at public health facilities of Gamo Gofa Zone, Southern, Ethiopia.

\section{Materials and Methods}

\section{Study setting}

Study was conducted in public health institutions of Gamo Gofa Zone which is located about $505 \mathrm{kms}$ South West of Addis Ababa, and about $275 \mathrm{~km}$ from Hawassa, the capital of the Southern Ethiopian. It has 15 Woredas and 2 Town administrations: within these there are 3 hospitals and 68 health centers. Facility based cross-sectional study design with both quantitative and qualitative methods of data collection was conducted from January 1 to February 28, 2015. 


\section{Study population}

All public health facilities in Gamo Gofa Zone currently treating STIs, health professionals working there and STI patients in catchment area of these public health facilities.

\section{Sample size and sampling techniques}

Sample size was calculated using stat calc program of Epi Info and considering the following assumptions: 18\% (knowledge of health care provider), ${ }^{16} 95 \%$ confidence level and $5 \%$ level of precision. Then by adding $10 \%$ non-responses rate, the final sample size was found to be 250 . For observation, 12 health institutions $\& 120$ STI patient cards were observed and 12 heads of respective health institutions were interviewed. And for mystery clients, total of 26 clients were hired. Of the health facilities in the Zone, 2 hospitals and 10 health centers were selected by SRS technique and sample size was proportionally allocated to health institutions. Then by using cluster sampling all eligible health professionals in the selected health institutions were selected. For mystery client interview, 3 clients/hospital and 2 clients/ health centers were interviewed.

\section{Data collection instrument and procedures}

For observation, the data were collected using District STI quality of Care Assessment (DISCA) tool ${ }^{17,18}$ and 12 heads of the health facilities have responded to it. For knowledge assessment, self administered questionnaire which is part of DISCA and for mystery clients, adapted semi structured questionnaire was used.

\section{Data analysis}

The data were coded and entered into EPI info version 7.0.1 and data was exported to SPSS version 20 for analysis. Descriptive statistics, vicariate and multivariate analysis was done using binary logistic regressions. Statistical significance was set at $\mathrm{P}<0.05$ and adjusted odds ratio was computed to assess the association between explanatory and outcome variables.

\section{Ethical considerations}

Ethical clearance was obtained from ethical review committee of College of Medicine and Health Sciences, Arba Minch University. Permission to conduct the study was also sought from the administrative authority of the respective health institutions. Before enrollment, informed written and verbal consent was taken from respondents.

\section{Results}

\section{Socio demographic characteristics}

The overall response rate was $100 \%$. Regarding clinicians, 174(69.6\%) were Diploma Nurses, 142(56.8\%) were male and More than half of the clinicians, 141(56.4\%) were below the age of 30years and only quite a small proportion $15(6 \%)$ were at the age of 50 and above. $50 \%$ of the clinicians have a median of sixyears of experience (Table 1).

\section{Structure}

\section{Examination facilities}

All 12 health facilities have examination coach and only 1 health center has examination light in consultation room which itself was not functional. None of health facilities has vaginal specula in consultation rooms. 10 out of 12 health facilities have examination gloves in consultation rooms. There were no National STI Syndromic management guidelines in health facilities. Client education materials about STI/HIV prevention and treatment were available only in 2 health facilities and were not written in local language. The health centers have on average 3 adult consultation rooms and the hospitals have 3 and 4 adult consultation rooms.

Table I Socio demographic characteristics of clinicians in public health facilities of Gamo Gofa Zone, Southern Ethiopia, Jan I to Feb 28, 2015

\begin{tabular}{|c|c|c|}
\hline Variables & $\begin{array}{l}\text { Number } \\
(n=250)\end{array}$ & $\begin{array}{l}\text { Percentage } \\
\text { (\%) }\end{array}$ \\
\hline \multicolumn{3}{|l|}{ Profession } \\
\hline Diploma Nurse & 174 & 69.6 \\
\hline BSc Nurse & 22 & 8.8 \\
\hline Health officer & 42 & 16.8 \\
\hline Medical Doctor & 12 & 4.8 \\
\hline \multicolumn{3}{|l|}{ Sex } \\
\hline Male & 142 & 56.8 \\
\hline Female & 108 & 43.2 \\
\hline \multicolumn{3}{|l|}{ Age } \\
\hline Less than or equal to 29 & $|4|$ & 56.4 \\
\hline 30 to 39 & 70 & 28 \\
\hline 40 to 49 & 24 & 9.6 \\
\hline 50 to 59 & 13 & 5.2 \\
\hline 60 and above & 2 & 0.8 \\
\hline \multicolumn{3}{|l|}{ Mean $\pm S D(31.43 \pm 8.31)$} \\
\hline \multicolumn{3}{|l|}{ work experience } \\
\hline Less than Iyear & 5 & 2.0 \\
\hline I to 5years & 115 & 46.0 \\
\hline 6 to $10 y e a r s$ & 71 & 28.4 \\
\hline greater than I0years & 59 & 23.6 \\
\hline Median (6.0) & & \\
\hline
\end{tabular}

\section{Health personnel}

In health centers, the number of clinicians ranged from 17 to 41 with average of 31 clinicians in each health center and the total number of clinicians in 10 health centers was 312 where as the number of clinicians in Hospitals was 267. According to the heads, of the clinicians working in health facilities, only $15(4.8 \%)$ and $7(2.6 \%)$ were trained on Syndromic management of STIs in health centers and hospitals respectively. Of the total trained clinicians, $9(60 \%)$ and $3(42.9 \%)$ clinicians were working in health centers and hospitals respectively during the study time. But of 250 clinicians participated in the study, only 32(12.8\%) were trained on Syndromic management of STIs and $50 \%$ of these had received their training before a median of 4.5 years prior to this study.

\section{Knowledge of clinicians}

Of 250, clinicians who mentioned the name of the drug, dosage, frequency and duration correctly according to National guideline were for urethral discharge $68(27.2 \%)$, for vaginal discharge $48(19.2 \%)$, for genital ulcer $5(2 \%)$ and for pregnant women with vaginal discharge only $4(1.6 \%)$ (Table 2$)$. And finally clinicians who mentioned the name of the drug, dosage, frequency and duration correctly for the 
drug in place of doxycycline for discharge management were $75(30 \%)$ Further analysis to assess determinants of knowledge of urethral discharge shows that profession of clinicians and type of health facility they were working in were significant determinants (Table 3). Health officers have knowledge of urethral discharge 4times more likely than Diploma Nurses (AOR $=4.326 ; 95 \% \mathrm{CI}=2.019,9.268)$. Likewise the odds of having knowledge of urethral discharge for Medical Doctors was 8times higher than Diploma Nurses $(\mathrm{AOR}=7.788 ; 95 \% \mathrm{CI}=2.021$, 30.006). And clinicians working in Health Centers have knowledge of urethral discharge 5times more likely than clinicians working in Hospitals (AOR $=0.194 ; 95 \% \mathrm{CI}=0.092,0.412)$.

\section{STI drug availability}

It is all in Table 4

\section{Structural quality}

The above findings indicate that the structural quality in all 12 health facilities for treatment of STIs syndromically was poor.

Table 2 Clinicians knowledge of treatment for STI syndromes: trained and untrained in public health facilities of Gamo Gofa Zone, Southern Ethiopia, Jan I to Feb 28,2015

\begin{tabular}{llll}
\hline Knowledge of syndromes & Trained (32) & Untrained (2 I8) & Total \\
\hline $\begin{array}{llll}\text { Has knowledge of urethral discharge } \\
\text { Yes }\end{array}$ & $12(37.5 \%)$ & $56(25.7 \%)$ & $\mathbf{6 8}(\mathbf{2 7 . 2} \%)$ \\
No & $20(62.5 \%)$ & $162(74.3 \%)$ & $182(72.8 \%)$ \\
Has knowledge of vaginal discharge & & & \\
Yes & $5(15.6 \%)$ & $43(19.7 \%)$ & $\mathbf{4 8 ( 1 9 . 2 \% )}$ \\
No & $27(84.4 \%)$ & $175(80.3 \%)$ & $202(80.8 \%)$ \\
Has knowledge of genital ulcer & & & \\
Yes & $1(3.1 \%)$ & $4(1.8 \%)$ & $\mathbf{5 ( 2 . 0 \% )}$ \\
No & $31(96.9 \%)$ & $214(98.2 \%)$ & $245(98.0 \%)$ \\
Has knowledge of pregnant women with vaginal discharge & & \\
Yes & $2(6.2 \%)$ & $2(0.9 \%)$ & $\mathbf{4 ( 1 . 6 \% )}$ \\
No & $30(93.8 \%)$ & $216(99.1 \%)$ & $246(98.4 \%)$ \\
\hline
\end{tabular}

Table 3 Determinants of knowledge of urethral discharge in public health facilities of Gamo Gofa Zone, Southern Ethiopia, Jan I to Feb 28,20 I5

\begin{tabular}{|c|c|c|c|c|}
\hline Determinants & $\mathbf{N}(\%)$ & Have Knowledge N(\%) & $\begin{array}{l}\text { Crude odds ratio( } 95 \% \\
\mathrm{CI})\end{array}$ & Adjusted odds ratio( $(95 \% \mathrm{Cl})$ \\
\hline \multicolumn{5}{|c|}{ Profession of clinicians } \\
\hline Diploma Nurse & $174(69.6)$ & 39 (22.4\%) & 1 & 1 \\
\hline BSc Nurse & $22(8.8)$ & $2(9.1 \%)$ & $0.346(0.78, I .546)$ & $0.427(0.092,1.987)$ \\
\hline Medical Doctor & $12(4.8)$ & $5(4 \mid .7 \%)$ & $2.473(0.743,8.223)$ & $7.788(2.021,30.006)^{*}$ \\
\hline \multicolumn{5}{|c|}{ Type of health facility clinicians working in } \\
\hline Health center & $138(55.2 \%)$ & $52(37.7 \%)$ & 1 & 1 \\
\hline
\end{tabular}

*=significant results

Table 4 The drug supply for the syndromic management of STIs in I2 public health facilities of Gamo Gofa Zone, Southern Ethiopia, Jan I to Feb 28, 20I5

\begin{tabular}{|c|c|c|c|c|c|c|c|c|}
\hline \multirow{2}{*}{ Drug } & \multicolumn{2}{|c|}{ Currently in stock? } & \multicolumn{2}{|c|}{ Currently out of stock? } & \multicolumn{2}{|c|}{ In stock in last month } & \multicolumn{2}{|c|}{ Out of stock in last month } \\
\hline & Number & $\%$ & Number & $\%$ & Number & $\%$ & Number & $\%$ \\
\hline Ciprofloxacin & 12 & 100 & 0 & 0 & $\mathrm{II}$ & 91.7 & 1 & 8.3 \\
\hline Metronidazole & 12 & 100 & 0 & 0 & 12 & 100 & 0 & 0 \\
\hline Doxycycline & 12 & 100 & 0 & 0 & 12 & 100 & 0 & 0 \\
\hline Benzanthine Penicillin & $\mathrm{II}$ & 91.7 & $\mathrm{I}$ & 8.3 & $\mathrm{II}$ & 91.7 & 1 & 8.3 \\
\hline Spectinomycin & 8 & 66.7 & 4 & 33.3 & 8 & 66.7 & 4 & 33.3 \\
\hline Tetracycline & 6 & 50 & 6 & 50 & 5 & 41.7 & 7 & 58.3 \\
\hline Erythromycin & $\mathrm{II}$ & 91.7 & I & 8.3 & II & 91.7 & 1 & 8.3 \\
\hline Acyclovir & 8 & 66.7 & 4 & 33.3 & 8 & 66.7 & 4 & 33.3 \\
\hline
\end{tabular}




\section{Process}

\section{Services for STI patients}

All health facilities do not provide services for STI patients privately. In 11(91.7\%) of health facilities, RPR testing is done on site in the facility. In $7(58.3 \%)$ health facilities, there was no occasion over the last month condoms ran out and $8(66.7 \%)$ health facilities do not show STI clients how to use condom in their facility. $9(75 \%)$ health facilities have penile model for condom demonstration in their facility. Only $2(16.7 \%)$ health facilities have condoms put in some place in the compound of the facility for individuals who want to use it. $11(91.7 \%)$ health facilities do not have partner notification cards in their facility and only $1(8.3 \%)$ health facility has partner notification card. Concerning syphilis screening on pregnant mothers, in only $7(58.3 \%)$ health facilities it was done for all pregnant mothers who come to health facility for ANC first visit. Of the total reviewed 120 cards of STI patients, only in $10(8.3 \%)$ cards did the clinicians correctly followed the national guidelines and treated the patients correctly using the Syndromic approach.

\section{Process quality}

The above findings indicate that the process quality for treatment of STIs syndromically in all 12 health facilities was poor.

\section{Output}

Of 26 mystery clients, only 1(3.8\%) mystery client was asked to show the diseased part for physical examination. For them, the maximum waiting time was $3 \mathrm{hrs}$ and the minimum waiting time was 2 minutes. And $50 \%$ of clients have waited for 15 minutes. About the length of time they waited to the see the provider, 15(57.69\%) said not satisfied and the left $11(42.31 \%)$ said they were satisfied. On the other hand, the maximum time they spent with provider was 30 minutes and the minimum was 2 minutes. And the mean time they spent with provider was 15.46 minutes. When asked about the length of stay with the provider, $16(61.54 \%)$ said not satisfied and the other $10(38.46 \%)$ said satisfied. The number of clients greeted by the provider when arrived in consultation room was only $7(26.92 \%)$. In consultation room only $8(30.77 \%)$ clients were satisfied with the provider's behavior. And only 7(26.92\%) received advice. 20(76.92\%) said they were not satisfied with the privacy of the service. Only $8(30.77 \%)$ were told to bring their partner and 20(76.92\%) said they weren't asked by the provider to return for another visit. Regarding the overall satisfaction with the consultation session, majority $23(88.46 \%)$ said they were not satisfied (Figure $1 \& 2$ ). Concerning recommending the provider to a friend, majority $22(84.62 \%)$ said they will not recommend.

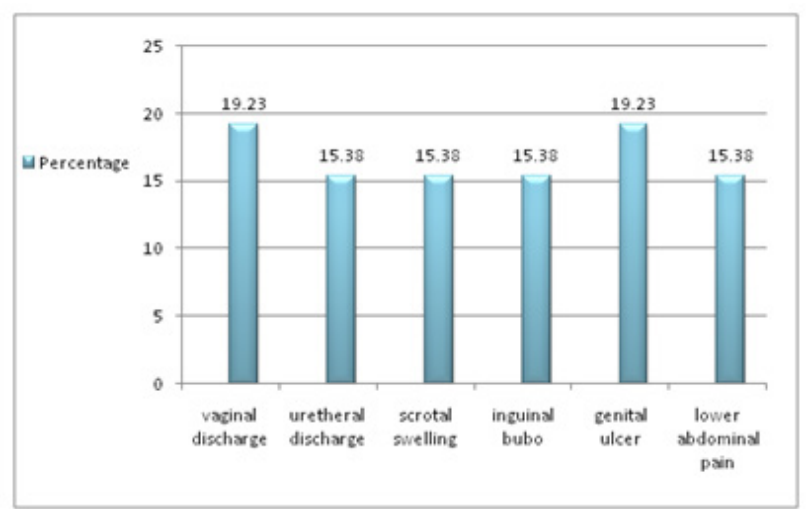

Figure I Syndromes mystery clients treated for in public health facilities of Gamo Gofa Zone, Southern Ethiopia, Jan I to Feb 28, 2015.

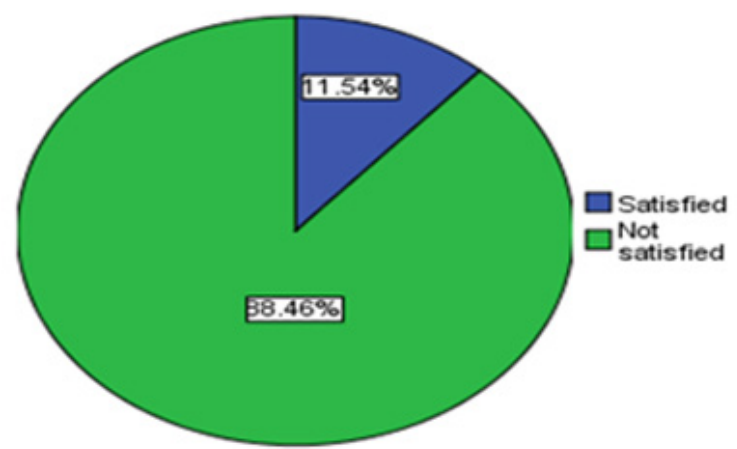

Figure 2 Overall satisfaction statuses of mystery clients in public health facilities of Gamo Gofa Zone, Southern Ethiopia, Jan I to Feb 28, 2015.

\section{Output quality}

The above findings indicate that output quality for management of STIs syndromically in all 12 health facilities was poor.

\section{Discussion}

\section{Structural quality}

According to HIV/AIDS Prevention and Control Office, In order to treat STI patients, National STI Syndromic management guidelines should be available in all public health facilities of Ethiopia. ${ }^{4}$ But findings of this study show that there were no guidelines in all health facilities. This is consistent with the finding of study conducted in Zaria, Northern Nigeria which showed no center had the Syndromic management manual or chart. ${ }^{19}$ However, this finding is far lower than that of the finding from study conducted in South Africa where 3 out of 4 Regional clinics have guideline in each consultation rooms. ${ }^{20}$ This may be attributed to the fact that South Africa has been working hardest to control STIs more. In order to follow the guideline and treat patients accordingly, clinicians have to have training on Syndromic management of STIs but in our study only $12.8 \%$ of clinicians were trained. This finding is higher than that of the finding from the study conducted in Windhoek, Namibia among private General Practitioners where none of them had specific training on Syndromic management. ${ }^{12}$ This could be attributed to our study was among clinicians working in public health facilities where government give priorities whereas the study in Namibia was among clinicians working in private health facilities. However, the finding from this study is completely below the finding of the study conducted in South Africa where all clinicians working in 3 out of 4 clinics were trained. ${ }^{20}$ In this study even if overall knowledge of clinicians varied by syndrome type it was all lowest that is the maximum knowledge the clinicians have was about treating urethral discharge that was $27.2 \%$. This result is lower than that of the study conducted on KAP of General Practitioners in Karachi, Pakistan and among private General Practitioners in Windhoek, Namibia where the knowledge for urethral discharge was $55.3 \%$ and $56.5 \%$ respectively. ${ }^{21,12}$ This might be because of these studies only focused on knowledge of General Practitioners while our study included lower level clinicians like Diploma Nurses also.

\section{Process quality}

In this study of the total reviewed 120 cards of STI patients, only in $10(8.3 \%)$ cards did the clinicians correctly followed the national guidelines and treated the patients correctly using the Syndromic approach.

This finding is almost consistent with the finding of the study conducted in Pakistan where most providers practiced personal 
empiricism..$^{22}$ However, this finding is slightly lower than the finding of the study conducted in six countries in West Africa where effective treatment was given to $14.1 \%$ of patients in conformity with national Syndromic STD management algorithms. ${ }^{23}$ This might attributable to the fact that this study covers the health facilities in six countries in West Africa which may include specialized health facilities while our study only included public health facilities in Gamo Gofa Zone of South Ethiopia. Similarly in the study, of 26 mystery clients, only $5(19.23 \%)$ were treated correctly according to the National guideline. When seen superficially, this finding seems to be a little bit higher than the finding of the study conducted in Pokhara, Nepal where chemists and druggists treated correctly according to guideline in $10.8 \%$ of simulated client cases ${ }^{24}$ but in fact chemists and druggists were not allowed to treat patients expect referring them to doctors. So the finding of the current study shows low performance according to national guide line.

\section{Output quality}

Of 26 mystery clients seen, only 1(3.8\%) of mystery client was asked to show the diseased part for physical examination. This finding is far behind the finding of the study conducted in six countries in West Africa where an adequate physical examination was carried out in $60.8 \%$ of cases. ${ }^{23}$ This might be because this study shows the findings from health care facilities in six West African countries while our study only shows the finding from public health facilities of a single Gamo Gofa Zone in South Ethiopia. In this study, of the mystery clients participated in the study, $57.7 \%$ were not satisfied with the length of time they waited to see the provider. This finding shows large number of clients not satisfied with the length of the time compared to the finding of the study conducted in Abidjan, Co ^te d'Ivoire where $44 \%$ of interviewed clients complained about a long waiting time. ${ }^{24}$ This might be attributed to the fact that since participants in study conducted in Co 'te d'Ivoire were female sex workers, in steady of complaining of the time they may shift to other health facilities where as the participants in current study were member of general population who may wait and complain of time rather than rushing to other health facilities. In this study the number of mystery clients greeted by the provider when arrived in consultation room was only $7(26.9 \%)$ and the number of those satisfied with the provider's behavior in consultation room was only $8(30.77 \%)$. This finding is far lower than the finding from the study conducted in Pokhara, Nepal where the providers talked with patient with a friendly face in $73 \%$ of simulated client, gave attention and showed interest in $83.8 \%{ }^{25}$ This might be because this study was done on health workers post training where as our study was done on health professionals randomly. In current study, of mystery clients seen in health facilities, only $23.1 \%$ were satisfied with the privacy of the service. This finding is similar with the finding of the study conducted in Pakistan where no public facility had enough space/privacy to effectively counsel clients on behavioral risk reduction..$^{22}$ In the study, of mystery clients seen in health facilities, only $30.77 \%$ were told to bring their partner. The finding is higher than the findings of the study conducted in Pakistan where Partner treatment was never discussed or administered ${ }^{22}$ and Windhoek Namibia where when managing a male patient on medical aid with urethral discharge for the first time, only $26 \%$ Doctors prompt partner notification. ${ }^{12}$ Whereas the finding is lower than the findings of the study conducted in Pokhara, Nepal where chemists and druggists notify the partner of the patient in $43.2 \%$ of simulated client ${ }^{24}$ and six countries in West Africa where patients were encouraged to advise their partners to seek treatment in $50.8 \%$ of cases. ${ }^{23}$ This might be because of the study in Pokhara, Nepal was done on health workers post training and for the study in six countries in West Africa, the study shows the findings from health care facilities in six West African countries. In this study, of mystery clients, only $11.54 \%$ were satisfied with the services and only $15.38 \%$ will recommend the provider to a friend. This finding is far lower than the finding of the study conducted in Abidjan, Co 'te d'Ivoire where most clients were happy with the clinic and with the attitude of the health care provider, and would return to the same place for the next health problem. ${ }^{25}$ This could be attributable to the fact that these health facilities in Abidjan, Co'ted'Ivoire were the facilities female sex workers chosen for themselves and made a regular customer where as in current study the mystery clients were gone to random health facilities.

\section{Conclusion}

In general the study indicated that quality of care for Sexually Transmitted Infections syndromic case management at public health facilities of Gamo Gofa Zone was poor which may be explained by the lack of National STI syndromic management guidelines, examination lights and vaginal specula in health facilities, inadequate knowledge of clinicians, inability of clinicians to treat STI patients correctly and unsatisfied clients.

\section{Acknowledgements}

Our sincere thank goes to Arba Minch University for provision of the opportunity to conduct the research. We also like to give our deepest gratitude for Gamo Gofa zone health departments for providing baseline information. Lastly our thanks go to data collectors and all research participants who took part in the study, without whom this research wouldn't have been in to existence.

\section{Conflict of interest}

Author declares that there is no conflict of interest.

\section{References}

1. Sexually transmitted infections. Geneva: World Health Organization; 2013.

2. Shrivastava SR, Shrivastava S, Ramasamy J. Utility of syndromic approach in management of sexually transmitted infections: public health perspective. Journal of Coastal Life Medicine. 2014;2(1):7-13.

3. 10 facts on sexually transmitted infections. Geneva: World Health Organization; 2013.

4. National Guidelines for the management of sexually transmitted infections using the syndromic approach. Addis Ababa: FMOH; 2006.

5. Global prevalence and incidence of selected curable sexually transmitted infections: overview and estimates. Geneva: World Health Organization; 2001.

6. White RG. Curable Sexually Transmitted Infection Treatment Interventions to Prevent HIV Transmission in Sub-Saharan Africa. The Open Infectious Diseases Journal. 2009;3:148-155.

7. Moges B, Yismaw G, Kassu A, et al. Sexually transmitted infections based on the syndromic approach in Gondar town, northwest Ethiopia: a retrospective study. BMC Public Health. 2013;13:143.

8. Global strategy for the prevention and control of sexually transmitted infections: 2006-2015. Geneva: World Health Organization; 2007. 
9. Taderera H. Global Policy on Sexually Transmitted Infections: Frameworks and interventions in Zimbabwe. Journal of Sociological Research. 2012;3(2).

10. Management of sexually transmitted infections using syndromic management approach. Guidelines for Service Providers. 3rd ed. Ministry of Health: Malawi. World health organization, National AIDS Commission, Geneva; 2007.

11. Smeltzer SC, Bare BG. Textbook of medical-surgical nursing. 9th ed. Lippincott, New York; 2000.

12. Iipinge SN, Pretorius L. The Delivery and Quality of Sexually Transmitted Infections Treatment by Private General Practitioners in Windhoek Namibia. Glob J Health Sci. 2012;4(5):156-171.

13. Vuylsteke B. Current status of Syndromic management of sexually transmitted infections in developing countries. Sex Transm Infect. 2004;80(5):333-334

14. Donabedian A. An Introduction to Quality Assurance in Health Care. New York: Oxford University; 2003.

15. Al-Assaf AF, Sheikh M. Quality improvement in primary health care: a practical guide. WHO Regional Publications, Eastern Mediterranean Series 26; 2004.

16. Khan MS, Unemo M, Zaman S, et al. Knowledge, Attitudes And Practices Regarding Human Immunodeficiency Virus/Acquired Immune Deficiency Syndrome And Sexually Transmitted Infections Among Health Care Providers In Lahore, Pakistan. J Ayub Med Coll Abbottabad. 2009;21(4):1-6.

17. Coetzee N, Magwaza S. District STD Quality of Care Assessment: guide lines for district clinic supervisor in using the 'DISCA' evaluation instrument. University of Cape Town, South Africa; 1998.
18. Moys A. A practical guide to using the District STI Quality of Care Assessment (DISCA). Reproductive Health Research Unit, Witwatersrand University, South Africa; 2002.

19. Olayinka AT, Shehu AU. An assessment of sexually transmitted infection management at the primary health care level in a local government in Northern Nigeria. International Journal of Medicine and Public Health. 2011;1(2):67-72

20. Moys A. Evaluating quality of STI management at a Regional Level using District STI Quality of Care Assessment Tool (DISCA). Reproductive Health Research Unit, Witwatersrand University, South Africa; 2002.

21. Hussain MF, Khanani MR, Siddiqu SE, et al. Knowledge, Attitudes \& Practices (KAP) of General Practitioners (GPs) regarding Sexually Transmitted Diseases (STDs) and HIV/AIDS in Karachi, Pakistan. J Pak Med Assoc. 2011;61(2):202-205.

22. Khan AA, Khan A. Sexually transmitted infection care in Pakistan: The providers perspective. J Pak Med Assoc. 2012;62:941.

23. Bitera R, Alary M, Mâsse B, et al. Quality of diseases management of sexually transmitted diseases: investigation of care in six countries in West African. PubMed. 2002;12(2):233-239.

24. Khan MMH, Wolter S, Mori M. Post-training quality of syndromic management of sexually transmitted infections by chemists and druggists in Pokhara, Nepal: is it satisfactory? International Journal for Quality in Health Care. 2006;18(1):66-72.

25. Vuylsteke B, Traore M, Mah-Bi G, et al. Quality of sexually transmitted infections services for female sex workers in Abidjan. Co 'te d'Ivoire. 2004;9(5):638-643. 